\section{CAMPAIGN PROVIDES TOOTHBRUSHING GUIDANCE FOR KIDS}

Henry Schein Dental UK is supporting BrushUpUK's free oral health educational programme by donating toothbrushing guidance booklets for 1,500 children. The booklets are part of Ian Aguado-Bush's Clean My Teeth Campaign to improve children's oral health and have been designed in close consultation with the British Dental Health Foundation. They are specially made to engage younger readers and guide them through the correct method of brushing their teeth, together with information on how food and drink affects their oral health.

BrushUpUK is a free oral health educational programme, founded in January 2015. A team of BrushUpUK qualified dental care professionals (DCPs) provide tailored presentations to schools, SEN schools, care homes and palliative care units in the local area, delivering practical, sensory and visual displays. These strive to improve the oral hygiene and attitude towards oral hygiene of their clients, staff and students.

As part of BrushUpUK primary school oral health presentations, the children get their own new toothbrush from the British Dental Health Foundation.

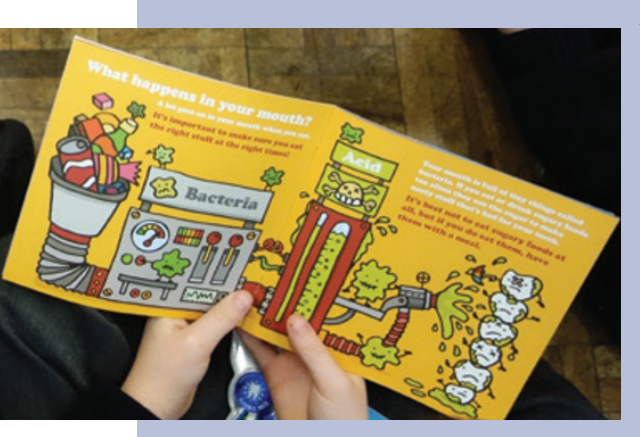
Afterwards, the lessons focus on the children using their new toothbrush to brush their teeth correctly. At the end of each presentation, the children get the Clean My Teeth Campaign books, donated by Henry Schein, to enforce what they have learnt with BrushUpUK, regarding healthy food choices and tooth brushing.

In the first 11 months of initial operation BrushUpUK presented to 865 children ranging from three to 16 years, 270 of these from special educational need schools.

www.brushupuk.com, www.cleanmyteeth.co.uk

\section{THE IMPACT OF DENTINE HYPERSENSITIVITY}

GSK Consumer Healthcare, manufacturers of Sensodyne, hosted a special symposium at the FDI conference in Bangkok to share results of research into the impact of dentine hypersensitivity (DH).

The research used the Dentine Hypersensitivity Evaluation Questionnaire (DHEQ), a validated tool, in order to demonstrate the impact of DH., ${ }^{1,2}$ Through a new pooled analysis of DHEQ data, Sensodyne revealed some surprising findings about how people who experience the condition will compensate, cope and alter their social activities when living with dentine hypersensitivity:3,4

- Nine out of ten sufferers had been experiencing the condition for longer than one year

- $70.4 \%$ of DH sufferers considered their sensitivity takes a lot of pleasure out of eating and drinking

- 59.4\% of DH sufferers try to avoid the sensations by biting food into small pieces.

Research also confirmed the clinical benefits of continuous twice-daily use of sensitivity toothpaste. ${ }^{3,4}$

1. Boiko O V Baker S R, Gibson B J et al. Construction and validation of the quality of life measure for dentine hypersensitivity (DHEQ). J Clin Periodonto/ 2010; 37: 973-980.

2 Baker S R, Gibson B J, Sufi F, Barlow A, Robinson P G. The Dentine Hypersensitivity Experience Questionnaire: a longitudinal validation study. J Clin Periodonto/2014; 41: 52-59.

GSK Data on File RH02026

4. Sufi F, Baker $S$. The subjective experience of dentine hypersensitivity - a pooled analysis. Presented at the 93rd General Session \&t Exhibition of the IADR, 2015.

\section{CITRUS AND PEPPERMINT FLAVOUR GLOVES}

Unigloves has more than 13 years' experience in the UK dental industry and has just launched its latest range of gloves: Vitality.

The Vitality range of gloves includes:

- Vitality Nitrile - white, powder-free with Lano-E coating

- Vitality Latex - white, powder-free with Lano-E coating

- Vitality Latex scented - green, powder-free with Lano-E coating and scented with a pleasant citrus and peppermint flavour for your patients' comfort.

The lanolin and vitamin E (Lano-E) coating is designed to reduce skin irritation and dehydration. This is supported by the multiple wash cycle which makes the gloves cleaner and safer by reducing residual water-soluble proteins and other harmful chemicals, thereby minimising allergy risks.

The double-chlorinated beaded cuffs facilitate easy donning and removal of gloves whilst the micro-roughened texture ensures optimal grip. This unique combination of features makes the Vitality range of gloves ideal for use within the dental industry.

For further information about Unigloves products, visit www.unigloves.co.uk or call 01634726516.

\section{LEARN MORE ABOUT ENHANCED CPD}

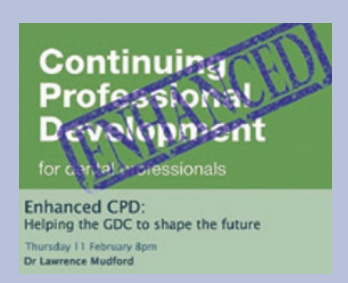

The GDC Enhanced CPD scheme is scheduled for introduction next year, so if you missed the GDC live webinar on The Dental Channel earlier this month and want to know what it will involve you can still view the recording online at your leisure.

Dr Lawrence Mudford (GDP, GDC Council member and Regional Dental Tutor for Health Education: Kent, Surrey and Sussex Deanery) covers the changes that are proposed for recording CPD, exploration of using CPD to enhance patient care, and what is involved in participating in the enhanced CPD pilot scheme.

The Enhanced CPD scheme will directly affect all dentists and dental care professionals, so this is an excellent opportunity to find out what is involved and make your voice heard.

This is free for Premium members of The Dental Channel. Those who do not hold a Premium membership can enrol for $£ 15$. A certificate for one hour of verifiable CPD will be provided on completion.

For further details and to enrol see http://bit.ly/ GDC-LW or call The Dental Channel on 02082999742. 